\title{
On the Power Series Coefficients of the Riemann Zeta Function
}

\author{
Yasushi MATSUOKA \\ Shinshu University \\ (Communicated by S. Koizumi)
}

\section{§1. Introduction and the main result.}

The Laurent expansion of the Riemann zeta function $\zeta(s)$ about the pole can be written in the form, in [2],

$$
\zeta(s)=\frac{1}{s-1}+\sum_{n=0}^{\infty} \frac{(-1)^{n}}{n !} \gamma_{n}(s-1)^{n}
$$

with

$$
\gamma_{n}=\lim _{N \rightarrow \infty}\left(\sum_{k=1}^{N} \frac{\log ^{n} k}{k}-\frac{\log ^{n+1} N}{n+1}\right) .
$$

Here $\log ^{0} k$ mean 1 for all $k$ including $k=1$. $\gamma_{0}$ is the well known Euler constant, and, for $n \geqq 1, \gamma_{n}$, sometimes called generalized Euler constants, have been studied by many authors ([1], Entry 13; or [3], p. 51). In this paper we shall give an asymptotic expansion of $\gamma_{n}$ for arbitrary large $n$, which yields some interesting results on $\gamma_{n}$. They can be found in [4].

We begin by defining some notations. Let $N$ be a nonnegative integer, and let $n$ be a positive integer. In order to write our theorem, we need two functions $a=a(n)$ and $b=b(n)$ which are given by the following lemma.

Lemma 1. If $n>c_{1}$, where $c_{1}$ is a sufficiently large constant, then the system of the equations

$$
\begin{aligned}
& -(n+1) \frac{y}{x^{2}+y^{2}}+\frac{1}{2} \pi-\operatorname{Im} \psi(x+i y)=0, \\
& -(n+1) \frac{x}{x^{2}+y^{2}}-\log 2 \pi+\operatorname{Re} \psi(x+i y)=0,
\end{aligned}
$$

with unknown $x$ and $y$, satisfying $0<y<x$ and $n^{1 / 2}<x<n$, has a unique $\overline{\text { Received February }}$ 4, 1988 
solution $x=a, y=b$, where $\psi(y)$ is the logarithmic derivative of the gamma function, i.e., $\psi(z)=\Gamma^{\prime}(z) / \Gamma(z)$, and $\operatorname{Re} z$ and $\operatorname{Im} z$ mean the real and imaginary parts of $z$, respectively.

For given $n$ with $n>c_{1}$, the pair $x=a, y=b$ is uniquely determined by the lemma. Hence these $a$ and $b$ can be considered as the functions of $n$, so that we denote these new functions by $a=a(n)$ and $b=b(n)$. We next define the functions $\phi(z), g(y), f(y)$ by

$$
\begin{aligned}
& \phi(z)=-(n+1) \log z-z \log (2 \pi i)+\log \Gamma(z), \\
& g(y)=\operatorname{Re} \phi(a+i y) \text { and } f(y)=\operatorname{Im} \phi(a+i y)
\end{aligned}
$$

with a real variable $y$, namely,

$$
\begin{aligned}
& g(y)=-\frac{1}{2}(n+1) \log \left(a^{2}+y^{2}\right)-a \log 2 \pi+\frac{1}{2} \pi y+\operatorname{Re} \log \Gamma(a+i y), \\
& f(y)=-(n+1) \arctan \left(\frac{y}{a}\right)-y \log 2 \pi-\frac{1}{2} \pi a+\operatorname{Im} \log \Gamma(a+i y) .
\end{aligned}
$$

Moreover, define the sequences $h_{k}, u_{k}, v_{k}(k=0,1,2,3, \cdots)$ by

$$
\begin{aligned}
\sum_{k=0}^{\infty} h_{k}(y-b)^{k}= & \exp \left\{\phi(a+i y)-\phi(a+i b)+\frac{1}{2} \phi^{\prime \prime}(a+i b)(y-b)^{2}\right\}, \\
& u_{k}=\operatorname{Re} h_{k} \text { and } v_{k}=\operatorname{Im} h_{k},
\end{aligned}
$$

then we have the following theorem which states the asymptotic expansion of $\gamma_{n}$.

THEOREM. Let $N$ be a nonnegative integer. If $n>c_{2} \Gamma(N / 3+17 / 6)$, where $c_{2}$ is a sufficiently large constant, then

$$
\begin{aligned}
\gamma_{n}=\frac{1}{\pi} & n ! e^{g(b)} \sum_{k=0}^{N}\left|h_{2 k}\right| 2^{k+1 / 2} \Gamma\left(k+\frac{1}{2}\right)\left\{g^{\prime \prime}(b)^{2}+f^{\prime \prime}(b)^{2}\right\}^{-k / 2-1 / 4} \\
& \times \cos \left\{f(b)-\left(k+\frac{1}{2}\right) \arctan \left(\frac{f^{\prime \prime}(b)}{g^{\prime \prime}(b)}\right)+\arctan \left(\frac{v_{2 k}}{u_{2 k}}\right)\right\} \\
& +O\left\{\Gamma\left(N+\frac{3}{2}\right) 2^{N} n ! e^{g(b)} n^{-N / 3+1 / 8} \log ^{2 N / 3-1 / 3} n\right\} .
\end{aligned}
$$

\section{§2. Proof of Lemma 1 and some other lemmas.}

Proof of Lemma 1. The equations (2) and (3) are the imaginary and real parts of the equation 


$$
\phi^{\prime}(x+i y)=-\frac{n+1}{x+i y}-\log (2 \pi i)+\psi(x+i y)=0,
$$

respectively. Hence we will show that the equation $z \phi^{\prime}(z)=0$ with $z=$ $x+i y$ has a unique solution under the assumption of the lemma. Now, we put

$$
h(y)=\operatorname{Im} z \phi^{\prime}(z)=-y \log 2 \pi-\frac{1}{2} \pi x+\operatorname{Im}(x+i y) \psi(x+i y)
$$

Then, if we use the asymptotic expansion of $\psi(z)$ which is derived from the asymptotic expansion of $\log \Gamma(z)$ ([6], p. 251), we can prove that, for sufficiently large $x, h(y)$ is steadily increasing in $0 \leqq y \leqq x$, having the values $h(0)<0$ and $h(x)>0$. Therefore the equation $h(y)=0$ has a unique solution, say, $y_{x}$, in $0<y<x$. We further set $z_{x}=x+i y_{x}$ and define

$$
\begin{aligned}
u(x)=\operatorname{Re} z_{x} \phi^{\prime}\left(z_{x}\right) & =-(n+1)+x \log 2 \pi+\frac{1}{2} \pi y_{x}+\operatorname{Re}\left(x+i y_{x}\right) \psi\left(x+i y_{x}\right) \\
& =-n-1+\left(x+\frac{y_{x}}{x}\right)\left(-\log 2 \pi+\operatorname{Re} \psi\left(x+i y_{x}\right)\right)
\end{aligned}
$$

Then, using the asymptotic expansion of $\psi(z)$, we can prove that, for sufficiently large $n, u(x)$ is steadily increasing in $n^{1 / 2} \leqq x \leqq n$, having the values $u\left(n^{1 / 2}\right)<0$ and $u(n)>0$. Hence the equation $u(x)=0$ has a unique solution, say $a$, in $n^{1 / 2}<x<n$. It follows that $z=a+i y_{a}$ is the unique solution of $z \phi^{\prime}(z)=0$. This completes the proof.

LEMMA 2. For sufficiently large $n$,

$$
n \log ^{-1} n<a<n \log ^{-1} n+2 n \log ^{-2} n \log \log n,
$$

and

$$
b=\frac{1}{2} \pi n \log ^{-2} n+O\left(n \log ^{-3} n \log \log n\right) .
$$

Proof. We have from (5)

$$
-b \log 2 \pi-\frac{1}{2} \pi a+\operatorname{Im}(a+i b) \psi(a+i b)=0 .
$$

Using (9), and $\psi(z)=\log z-z^{-1} / 2+O\left(|z|^{-2}\right)$, we have $b / a<2 \log ^{-1} a$, and further

$$
\frac{b}{a}=\frac{1}{2} \pi \log ^{-1} a+O\left(\log ^{-2} a\right)
$$


It follows from (6) that

$$
\begin{aligned}
n & =-1+\left(a+\frac{b}{a}\right)(-\log 2 \pi+\operatorname{Re} \psi(a+i b)) \\
& =a \log a-a \log 2 \pi+O\left(a \log ^{-1} a\right) .
\end{aligned}
$$

Here we suppose that $a \leqq n \log ^{-1} n$. Then we have from (11) $n<$ $a \log a \leqq n \log ^{-1} n \log \left(n \log ^{-1} n\right)<n$ for sufficiently large $n$. This is a contradiction, and hence $a>n \log ^{-1} n$. We next suppose that $a \geqq n \log ^{-1} n+$ $2 a \log ^{-2} n \log \log n$. Then, by (11), we have $n>a \log a-2 a \geqq n \log ^{-1} n(1+$ $\left.2 \log ^{-1} n \log \log n\right) \log \left\{n \log ^{-1} n\left(1+2 \log ^{-1} n \log \log n\right)\right\}-2 n \log ^{-1} n\left(1+2 \log ^{-1} n \times\right.$ $\log \log n)>n$. This is a contradiction, and hence $a<n \log ^{-1} n+2 \log ^{-2} n \times$ $\log \log n$, which completes (7). Moreover, we can obtain (8) from (7) and (10).

LEMMA 3. $g(y)$ is steadily increasing in $0 \leqq y \leqq b$, has the maximum at $y=b$, and is steadily decreasing in $b \leqq y \leqq a$, having the properties $g(b)-g(b-\Delta)>(1 / 3) \log ^{3} a, \quad g(b)-g(b+\Delta)>(1 / 3) \log ^{3} a$, where $\Delta=a^{1 / 2} \log a$; further, $g^{\prime \prime}(b)=-a^{-1} \log a+(\log 2 \pi-1) a^{-1}+O\left(a^{-1} \log ^{-1} a\right)$, and $f^{\prime \prime}(b)=$ $a^{-1} \pi+O\left(a^{-1} \log ^{-1} a\right)$.

Proof. Using the asymptotic expansion of $\log \Gamma(z)$, we have, for $0 \leqq y \leqq a$,

$$
g^{\prime \prime}(y)=-\left(n+\frac{3}{2}\right) \frac{a^{2}-y^{2}}{\left(a^{2}+y^{2}\right)^{2}}-\frac{a}{a^{2}+y^{2}}+O\left(a^{-3}\right)<0
$$

It follows that $g^{\prime}(y)$ is steadily decreasing in $0 \leqq y \leqq a$. Moreover, $g^{\prime}(0)>0$ and $g^{\prime}(a)<0$ for sufficiently large $n$, which leads to that $g(y)$ is steadily increasing in $0 \leqq y \leqq b$, has the maximum at $y=b$, and is steadily decresing in $b \leqq y \leqq a$, since $g^{\prime}(b)=0$.

Let $\delta=(\pi / 2) \log ^{-1} a$. Then $a+i b=a(1+i \delta)(1+O(\delta))$. It follows that

$$
\begin{aligned}
\phi^{\prime \prime}(a+i b) & =\left(n+\frac{3}{2}\right) a^{-2}(1+i \delta)^{-2}+a^{-1}(1+i \delta)^{-1}+O\left(a^{-1} \delta\right) \\
& =a^{-1}(\log a-\log 2 \pi)(1-2 i \delta)+a^{-1}+O\left(a^{-1} \delta\right)
\end{aligned}
$$

Hence, we obtain $g^{\prime \prime}(b)=-a^{-1} \log a+(\log 2 \pi-1) a^{-1}+O\left(a^{-1} \delta\right)$ and $f^{\prime \prime}(b)=$ $a^{-1} \pi+O\left(a^{-1} \delta\right)$

Now, if we use Taylor's theorem ([6], p. 96), then we have

$$
g(b+\Delta)=g(b)+\frac{1}{2} g^{\prime \prime}(b) \Delta^{2}+\frac{1}{6} g^{\prime \prime \prime}(\xi) \Delta^{3}
$$


with $b<\xi<b+\Delta$. Using (12), and

$$
g^{\prime \prime \prime}(\xi)=-\left(n+\frac{3}{2}\right) \frac{2 \xi^{5}-4 a^{2} \xi^{3}-6 a^{4} \xi}{\left(a^{2}+\xi^{2}\right)^{4}}+\frac{2 a \xi}{\left(a^{2}+\xi^{2}\right)^{2}}+O\left(a^{-4}\right),
$$

we have

$$
g^{\prime \prime \prime}(\xi)<12\left(n+\frac{3}{2}\right) a^{-3}+2 a^{-2}+O\left(a^{-4}\right)<12 a^{-2} \log a .
$$

It follows that

$$
g(b+\Delta)-g(b)<-\frac{3}{8} \Delta^{2} a^{-1} \log a+2 \Delta^{3} a^{-2} \log a<-\frac{1}{3} \log ^{3} a,
$$

since $g^{\prime \prime}(b)<-(3 / 4) a^{-1} \log a$. In case $g(b)-g(b-\Delta)$, we have the same result as well, which completes the proof.

LEMMA 4. For $j \geqq 0,\left|h_{j}\right| \leqq\left(a^{-1} \log a\right)^{2 j / 3}$.

Proof. We first prove that, if $p_{j}(j=0,1,2,3, \cdots)$ is the power series coefficients defined by $\phi(a+i y)=\sum_{j=0}^{\infty} p_{j}(y-b)^{j}$, then, for $j \geqq 2$,

$$
\left|p_{j}\right| \leqq \frac{1}{j}\left(a^{-1} \log a\right)^{j-1}
$$

In case $j \geqq 3$, differentiating $\phi(a+i y)$ two times by $y$, we have

$$
j(j-1) p_{j}=-\frac{1}{2 \pi i} \int_{C} \phi^{\prime \prime}(a+i w)(w-b)^{-j+1} d w,
$$

where $C$ is the circle with center $b$ and radius $a / 2$ in the positive direction. We have, on $C, \min |a+i w|=a / 2+O\left(a \log ^{-2} a\right)$, and $\left|\phi^{\prime \prime}(a+i w)\right|<$ $5 a^{-1} \log a$. Therefore, for $j \geqq 3$,

$$
\left|p_{j}\right|<\frac{1}{j}\left(a^{-1} \log a\right)^{j-1} \frac{5}{j-1}\left(2 \log ^{-1} a\right)^{j-2}<\frac{1}{j}\left(a^{-1} \log a\right)^{j-1} .
$$

In case $j=2$, we have, by Lemma $3,\left|p_{2}\right|=(1 / 2)\left|g^{\prime \prime}(b)+i f^{\prime \prime}(b)\right|<(1 / 2) a^{-1} \log a$, which implies (13) for all $j \geqq 2$.

We next prove the inequality in the lemma. If we differentiate (4), write the power series expansion with the variable $y-b$, and equate the terms of order $0,1,2,3, \cdots$ of both sides, then we have the system of the following infinite set of equations;

$$
\begin{aligned}
& h_{j}=0 \quad(j=1,2), \\
& j h_{j}=\sum_{k=2}^{j-1}(k+1) p_{k+1} h_{j-1-k} \quad(j=3,4,5,6, \cdots) .
\end{aligned}
$$


In order to prove the lemma, we use the mathematical induction with respect to $j$. For $j=0,1,2$, the inequality holds, since $h_{0}=1, h_{1}=0$, and $h_{2}=0$. We thus suppose that the inequality holds for $j=0,1,2,3, \cdots, J-1$. Then we have from (13)

$$
\begin{aligned}
\left|J h_{J}\right| & =\left|\sum_{k=2}^{J-1}(k+1) p_{k+1} h_{J-1-k}\right| \leqq \sum_{k=2}^{J-1}\left(a^{-1} \log a\right)^{k}\left(a^{-1} \log a\right)^{2(J-1-k) / 3} \\
& <\left\{1-\left(a^{-1} \log a\right)^{1 / 3}\right\}^{-1}\left(a^{-1} \log a\right)^{2 J / 3}<J\left(a^{-1} \log a\right)^{2 J / 3},
\end{aligned}
$$

since $\left\{1-\left(a^{-1} \log a\right)^{1 / 3}\right\}^{-1}<3$ for sufficiently large $a$, which implies that the inequality holds for $j=J$. This completes the proof.

\section{§3. Proof of Theorem.}

In this section we use Vinogradov's symbol «([3], p. XVI). A key idea in our proof is the fact that we can apply the saddle point method to our integral expression of $\gamma_{n}$. Here, we shall prove that $\gamma_{n}=I_{1}+I_{2}+$ $I_{3}+I_{4}+I_{5}+I_{8}+I_{7}+I_{8}+J_{9}$, I's are the error terms, and $J_{9}$ is the main term.

We first show

$$
\begin{aligned}
\gamma_{n}= & \frac{1}{\pi} n ! \operatorname{Re} \int_{0}^{\infty}(a+i y)^{-n-1}(2 \pi)^{-a-i y} e^{-\pi y / 2+\pi i a / 2} \Gamma(a+i y) \zeta(a+i y) d y \\
& +\frac{1}{\pi} n ! \operatorname{Re} \int_{0}^{\infty}(a+i y)^{-n-1}(2 \pi)^{-a-i y} e^{\pi y / 2-\pi i a / 2} \Gamma(a+i y) \zeta(a+i y) d y \\
= & I_{1}+J_{2},
\end{aligned}
$$

say. We have from (1)

$$
\gamma_{n}=\frac{n !}{2 \pi i} \int_{C} z^{-n-1} \zeta(1-z) d z
$$

where $C$ is a contour enclosing 0 . Using the order of $\zeta(s)$ ([5], pp. 81-82), we can deform $C$ into the line from $a-i \infty$ to $a+i \infty$. Moreover, using the functional equation of $\zeta(s)$ ([5], p. 13), and changing the variable $z$ to $y$ with $z=a+i y$, we have

$$
\gamma_{n}=\frac{n !}{2 \pi} \int_{-\infty}^{\infty}(a+i y)^{-n-1} 2(2 \pi)^{-a-i y} \cos \left\{\frac{1}{2} \pi(a+i y)\right\} \Gamma(a+i y) \zeta(a+i y) d y .
$$

We now divide the integral into two parts; $\int_{-\infty}^{0}+\int_{0}^{\infty}$. If we notice that the first intergal is the complex conjugate of the second, we obtain (14), using $2 \cos \{\pi(a+i y) / 2\}=\exp (-\pi y / 2+\pi i a / 2)+\exp (\pi y / 2-\pi i a / 2)$. 
We next prove

$$
I_{1} \ll n ! e^{g(b)} n^{-N / 8-1 / 3} \log ^{N / 3+1 / 3} n .
$$

Using asymptotic expansion of $\log \Gamma(a+i y)$, we have

$$
\begin{aligned}
I_{1} & \ll n ! \int_{0}^{\infty}\left(a^{2}+y^{2}\right)^{-(n-a+3 / 2) / 2}(2 \pi e)^{-a} e^{-\pi y / 2-y \arctan (y / a)} d y \\
& \ll n ! \exp \{-(n-a+1) \log a-a(\log 2 \pi+1)\} \ll n ! \exp \{g(0)\},
\end{aligned}
$$

since $g(0)=-(n-a+3 / 2) \log a-a(\log 2 \pi+1)+O(1)$. It follows from Lemma 3 that

$$
I_{1} \ll n ! \exp \{g(b-\Delta)\} \ll n ! \exp \left\{g(b)-\frac{1}{3} \log ^{3} a\right\},
$$

where $\Delta=a^{1 / 2} \log a$. By Lemma 1 and Lemma 2, we can prove $\log ^{2} a<$ $N+4$, and hence we obtain (15).

We next divide $J_{2}$ into two parts;

$$
J_{2}=\frac{1}{\pi} n ! \operatorname{Re}\left(\int_{0}^{a}+\int_{a}^{\infty}\right)=J_{3}+I_{2},
$$

say. We have by Lemma 2 and Lemma 3

$$
I_{2} \ll n !(2 \pi e)^{-a} \int_{a}^{\infty}\left(a^{2}+y^{2}\right)^{-(n-a+8 / 2) / 2} e^{\pi y / 2-y \arctan (y / a)} d y
$$

$$
\ll n ! e^{g(b)} n^{-N / 3-4 / 3} \log ^{N / 3+4 / 3} n .
$$

We next divide $J_{3}$ into two parts;

$$
\text { - } J_{3}=\frac{1}{\pi} n ! \operatorname{Re}\left\{\int_{0}^{a} e^{\phi(a+i y)} d y+\int_{0}^{a} e^{\phi(a+i y)}(\zeta(a+i y)-1) d y\right\}=J_{4}+I_{3},
$$

say. If we use the estimate $\zeta(a+i y)-1=O\left(2^{-a}\right)$, then we obtain

$$
I_{3} \ll n ! e^{g(b)} n \log ^{-1} n \exp \left(-n \cdot \log ^{-1} n \log 2\right) .
$$

We further divide $J_{4}$ into three parts;

$$
J_{4}=\frac{1}{\pi} n ! \operatorname{Re}\left(\int_{0}^{b-4}+\int_{b-4}^{b+4}+\int_{b+4}^{a}\right)=I_{4}+J_{6}+I_{5},
$$

say. We then have by Lemma 3

$$
\int_{0}^{b-4} e^{\phi(a+i y)} d y \leqq \int_{0}^{b-4} e^{g(y)} d y<a e^{g(b-4)},
$$


so that

$$
I_{4} \ll n ! e^{g(b)} n^{-N / 3-1 / 3} \log ^{N / 3-1 / 3} n
$$

We have also

$$
I_{5} \ll n ! e^{g(b)} n^{-N / 3-1 / 3} \log ^{N / 3-1 / 3} n .
$$

We next consider $J_{\theta}$. We can write

$$
\begin{aligned}
J_{\theta}= & \frac{1}{\pi} n ! \operatorname{Re} \int_{b-\Delta}^{b+\Delta} \exp \left\{\phi(a+i b)-\frac{1}{2} \phi^{\prime \prime}(a+i b)(y-b)^{2}\right\} \\
& \times \exp \left\{\phi(a+i y)-\phi(a+i b)+\frac{1}{2} \phi^{\prime \prime}(a+i b)(y-b)^{2}\right\} d y .
\end{aligned}
$$

We know that $\phi(a+i y)$ has the nearest singularity at $y=i a$, so that the power series $\sum_{k=0}^{\infty} h_{k}(y-b)^{k}$ defined by (4) converges in $b-\Delta \leqq y \leqq b+\Delta$. We now divide the power series into two parts;

$$
\sum_{k=0}^{\infty} h_{k}(y-b)^{k}=\sum_{k=0}^{2 N+1} h_{k}(y-b)^{k}+U_{N}
$$

with

$$
U_{N}=\sum_{k=2 N+2}^{\infty} h_{k}(y-b)^{k}
$$

We thus have

$$
\begin{aligned}
J_{b}= & \frac{1}{\pi} n ! \operatorname{Re} \int_{b-\Delta}^{b+\Delta} \exp \left\{\phi(a+i b)-\frac{1}{2} \phi^{\prime \prime}(a+i b)(y-b)^{2}\right\} \sum_{k=0}^{2 N+1} h_{k}(y-b)^{k} d y \\
& +\frac{1}{\pi} n ! \operatorname{Re} \int_{b-\Delta}^{b+\Delta} \exp \left\{\phi(a+i b)-\frac{1}{2} \phi^{\prime \prime}(a+i b)(y-b)^{2}\right\} U_{N} d y=J_{7}+I_{6},
\end{aligned}
$$

say. We are going to estimate $I_{6}$. We have by Lemma 4

$$
\left|U_{N}\right| \leqq \sum_{k=2 N+2}^{\infty}\left|h_{k}\right||y-b|^{k}<\sum_{k=2 N+2}^{\infty}\left\{\left(a^{-1} \log a\right)^{2 / 3} \Delta\right\}^{k}<2\left\{\left(a^{-1} \log a\right)^{2 / 3} \Delta\right\}^{2 N+2},
$$

since $\left(a^{-1} \log a\right)^{2 / s} \Delta<1 / 2$. We have further, for $b-\Delta \leqq y \leqq b+\Delta,(1 / 2) g^{\prime \prime}(b) \times$ $(y-b)^{2}<0$, so that

$$
I_{b} \ll n ! \Delta e^{g(b)}\left\{\left(a^{-1} \log a\right)^{2 / 3} \Delta\right\}^{2 N+2} .
$$

Hence we get

$$
I_{6} \ll n ! e^{g(b)} n^{-N / 3+1 / 8} \log ^{11 N / 3+25 / 8} n .
$$


We next divide $J_{7}$ into three parts;

$$
J_{7}=\frac{1}{\pi} n ! \operatorname{Re} \sum_{k=0}^{2 N+1} h_{k} \exp \{\phi(a+i b)\}\left(\int_{-\infty}^{\infty}-\int_{b+4}^{\infty}-\int_{-\infty}^{b-\Delta}\right)=J_{9}+I_{7}+I_{8},
$$

say. We are going to estimate $I_{7}$. For all $k$, the integrals in $I_{7}$ are estimated by

$$
\ll \Delta^{k+1} \int_{1}^{\infty} \exp \left(-\frac{1}{3} \log ^{3} a y^{2}\right) y^{k} d y,
$$

since $g^{\prime \prime}(b)<-(1 / 3) a^{-1} \log a$. We can now write the integrand as

$$
\exp \left(-\frac{1}{6} \log ^{3} a y^{2}\right) \times \exp \left(-\frac{1}{6} \log ^{3} a y^{2}\right) y^{k} \text {. }
$$

We see that the second term has the maximum at $y=\left(3 k \log ^{-1} a\right)^{1 / 2}<1$. Hence the integrals are estimated by

$$
\begin{aligned}
& \ll \Delta^{k+1} \exp \left(-\frac{1}{6} \log ^{3} a\right) \int_{1}^{\infty} \exp \left(-\frac{1}{6} \log ^{3} a y^{2}\right) d y \\
& <\Delta^{k+1} \exp \left(-\frac{1}{6} \log ^{3} a\right) \int_{1}^{\infty} y \exp \left(-\frac{1}{6} \log ^{3} a y^{2}\right) d y \\
& =3 \Delta^{k+1} \exp \left(-\frac{1}{6} \log ^{8} a\right) \log ^{-3} a .
\end{aligned}
$$

It follows that

$$
I_{7} \ll n ! e^{g(b)} n^{-N / 3-5 / 8} \log ^{N / 3-7 / 8} n .
$$

We have also

$$
I_{8} \ll n ! e^{g(b)} n^{-N / 3-5 / 8} \log ^{N / 3-7 / 8} n .
$$

We finally consider $J_{\vartheta}$. If we notice that the integrals in $J_{\vartheta}$ are zeros for all odd $k$, we get

$$
J_{\theta}=\frac{1}{\pi} n ! \operatorname{Re} \sum_{k=0}^{N} h_{2 k} e^{\phi(a+i b)} \int_{-\infty}^{\infty} \exp \left\{-\frac{1}{2} \phi^{\prime \prime}(a+i b)(y-b)^{2}\right\}(y-b)^{2 k} d y .
$$

Here we change the variable $y$ to $x$ with $x=(y-b)^{2}$ in the integral, and recall the Euler's expression of $\Gamma(z)([6]$, p. 241). Then we see that the integral is expressed by

$$
\Gamma\left(k+\frac{1}{2}\right) 2^{k+1 / 2}\left\{g^{\prime \prime}(b)^{2}+f^{\prime \prime}(b)^{2}\right\}^{-k / 2-1 / 4} \exp \left\{-i\left(k+\frac{1}{2}\right) \arctan \left(\frac{f^{\prime \prime}(b)}{g^{\prime \prime}(b)}\right)\right\} .
$$


Hence we finally obtain

$$
\begin{aligned}
J_{\theta}= & \frac{1}{\pi} n ! e^{g(b)} \sum_{k=0}^{N}\left|h_{2 k}\right| 2^{k+1 / 2} \Gamma\left(k+\frac{1}{2}\right)\left\{g^{\prime \prime}(b)^{2}+f^{\prime \prime}(b)^{2}\right\}^{-k / 2-1 / 4} \\
& \times \cos \left\{f(b)-\left(k+\frac{1}{2}\right) \arctan \left(\frac{f^{\prime \prime}(b)}{g^{\prime \prime}(b)}\right)+\arctan \left(\frac{v_{2 k}}{u_{2 k}}\right)\right\} .
\end{aligned}
$$

If we take account of the fact that $\gamma_{n}=I_{1}+I_{2}+I_{3}+I_{4}+I_{5}+I_{6}+I_{7}+I_{8}+J_{9}$, we get the formula from (15), (16), (17), (18), (19), (20), (21), (22), and (23). Although the $O$-term in the theorem is sharper than what we get, we can obtain the theorem, taking $N+1$ instead of $N$, and estimating the last term in the sum, and the $O$-term. This completes the proof.

ACKNowledgment. The author would like to thank Professor T. Mitsui for giving him the new elegant proof of Lemma 1 and his helpful comments concerning the material of this article.

\section{References}

[1] B. C. BERndT and R. J. Evans, Chapter 7 of Ramanujan's second note book, Proc. Indian Acad. Sci. (Math. Sci.), 92 (1983), 67-96.

[2] W. E. BRIGGs, Some constants associated with the Riemann zeta function, Michigan Math. J., 3 (1955/56), 117-121.

[3] A. Ivić, The Riemann Zeta-Function, John Wiley, New York, 1985.

[4] Y. Matsuoka, Generalized Euler constants associated with the Riemann zeta function, Number Theory and Combinatorics Japan 1984, 279-295, World Scientific Publ., Singapore, 1985.

[5] E. C. Titchmarsh, The Theory of the Riemann Zeta-Function, Oxford Univ. Press, 1951.

[6] E.T. Whittaker and G.N. Watson, A Course of Modern Analysis, fourth ed., Cambridge Univ. Press, 1927.

Present Address:

Department of Mathematics, Faculty of Education, Shinshu University

Nishinagano, Nagano 380, Japan 